\title{
The Face of Tropical Sprue in 2010
}

\author{
Mashal Jatoi Batheja ${ }^{a} \quad$ Jonathan Leighton ${ }^{a} \quad$ Ainara Azueta $^{b}$ \\ Russell Heigh ${ }^{a}$ \\ aDepartment of Gastroenterology and Hepatology, Mayo Clinic, Scottsdale, Ariz., \\ USA; 'bepartment of Anatomic Pathology, Hospital Txagorritxu, Vitoria, Spain
}

\section{Key Words}

Tropical sprue $\cdot$ Celiac disease $\cdot$ Diarrhea $\cdot$ Weight loss · Vitamin B12 deficiency $\cdot$ Tropical countries · Post-infective malabsorption

\begin{abstract}
Tropical sprue (TS), although endemic in certain tropical regions of the world, is rarely seen in North America and Europe. However, in this era of globalization and worldwide travel, it is important for all clinicians to be aware of the possibility of TS in patients presenting with nonspecific, persistent gastrointestinal complaints like diarrhea and weight loss. The symptoms and histologic findings of TS can resemble and be confused with those of diseases seen more commonly in nontropical climates like celiac disease and small intestine bacterial overgrowth. Therefore, if the usual causes of persistent diarrhea are ruled out, keeping a high index of suspicion for TS in patients who have a travel history to one of the endemic regions is important.
\end{abstract}

\section{Case 1}

A 58-year-old female presented for a second opinion regarding her persistent diarrhea, bloating, and weight loss. She had been given the diagnosis of celiac disease in the past. The patient, a United States resident, had lived overseas and spent time in several countries including Singapore, Thailand, and Indonesia. She developed diarrhea two and half years prior to her consultation with us while she was still residing in Southeast Asia. An evaluation for infectious causes of diarrhea was done initially in Singapore, and although no specific organism was found, she was treated empirically with metronidazole twice with no improvement in symptoms. Also, while in Singapore a year and a half prior to presentation, she was evaluated with upper endoscopy and colonoscopy. The upper endoscopy biopsies demonstrated partial villous blunting. Blood tests revealed a positive antigliadin antibody but a negative antiendomysial antibody. Based on this she was given a diagnosis of celiac disease and placed on a gluten-free diet. She was adherent to the gluten-free diet, but despite the dietary restrictions she continued to have watery diarrhea and weight loss. Her weight was $38.5 \mathrm{~kg}$ (BMI 15) at the time of her initial visit with us.

Laboratory evaluation at our institution revealed macrocytic anemia (Hgb $10.1 \mathrm{~g} / \mathrm{dl}$, MCV 122), low folate $(2.8 \mu \mathrm{g} / \mathrm{l}$, normal $>3.5 \mu \mathrm{g} / \mathrm{l})$, low vitamin B12 (<70 ng/l, normal 180-914 ng/l), and hypokalemia (3.2 $\mathrm{mmol} / \mathrm{l}$, normal 3.8-4 mmol/l). Tissue transglutaminase antibody, endomysial antibody, gliadin 
IgG and IgA, and total IgA levels were all normal. A CT enterography examination was normal. Upper endoscopy revealed a few gastric antral erosions and the duodenum appeared normal. Gastric biopsy showed nonspecific foveolar hyperplasia and the duodenal biopsy showed partial villous atrophy, mild crypt hyperplasia, and increase in chronic inflammation in the lamina propria including numerous plasma cells and intraepithelial lymphocytes. These changes were nonspecific but were consistent with TS as well as celiac disease. Duodenal aspirates were negative for Giardia lamblia and bacterial overgrowth.

In light of her negative serologies and lack of response to a gluten-free diet, it was felt that celiac disease was unlikely. Given her travel history, our suspicion for TS was high and she was started on tetracycline $250 \mathrm{mg}$ four times a day and folic acid supplementation $5 \mathrm{mg}$ daily. Relatively quickly, over the next two days, the patient reported feeling much better. The diarrhea and bloating improved, appetite and energy level increased, and she began to gain weight. On a follow-up visit two months later, the patient was continuing to improve. The diarrhea was completely gone, and her weight had increased from 36 to $48 \mathrm{~kg}$. She completed a six-month course of tetracycline and her symptoms have not returned.

\section{Case 2}

A 68-year-old male presented to our institution complaining of persistent watery diarrhea and weight loss. He had recently returned from Lima, Peru where he had spent 18 months as a missionary. He recalled that one year into his stay, he developed intermittent watery, nonbloody diarrhea that was persistent. He was also experiencing fatigue, lethargy, and had a $15 \mathrm{~kg}$ weight loss. While in Peru, he was evaluated and told that his stool studies were negative for any specific infection and that blood tests showed a vitamin B12 deficiency. In Peru he was empirically treated for a parasitic infection. After his return to the United States, his diarrhea continued intermittently and he was frustrated by lack of energy and inability to gain weight.

The patient was then evaluated at our institution. Physical exam was notable for signs of weight loss. Laboratory was notable for a low vitamin B12 level (<70 ng/l, normal 180-914 ng/l), high methylmalonic acid $(9.38 \mu \mathrm{mol} / \mathrm{l}$, normal 0-4.0 $\mu \mathrm{mol} / \mathrm{l})$. His CBC was normal, as was his folate level, intrinsic factor and celiac serology including endomysial antibody, tissue transglutaminase antibody, and gliadin IgA, gliadin IgG, and total IgA levels. HLA typing was not consistent with celiac disease. A colonoscopy revealed normal mucosa with random biopsies showing mild nonspecific changes. An upper endoscopy showed a normal-appearing mucosa throughout. Random duodenal biopsies showed partial villous atrophy with increased surface epithelial lymphocytes (fig. 1). Duodenal aspirates were taken for bacterial overgrowth and grew over 100,000 colony-forming units/mm of aerobic mixed Gram-positive and -negative organisms.

The differential diagnosis in this patient included small intestine bacterial overgrowth and TS. The patient was initially treated for the possibility of small intestinal bacterial overgrowth with metronidazole $500 \mathrm{mg}$ three times daily for two weeks. He was also initiated on weekly vitamin B12 injections. However, after completion of the metronidazole, the patient felt no improvement and he continued to have 3-5 loose stools daily. A glucose-hydrogen breath test for bacterial overgrowth was performed and was negative. Although small intestinal bacterial overgrowth could cause this constellation of signs and symptoms, he had no symptomatic improvement after treatment with metronidazole and a hydrogen breath test post-treatment was negative, suggesting ongoing symptoms despite eradication were due to something else. Therefore, given his stay in South America, vitamin B12 deficiency, and duodenal biopsies showing partial villous atrophy, TS was seriously considered. However, the patient was reluctant to start on a prolonged antibiotic course for TS and elected several months of observation.

At his follow-up visit a few months later, his symptoms of diarrhea, lethargy and inability to gain weight persisted. An upper endoscopy was repeated and again revealed normal mucosa but histologically there was persistence of small bowel partial villous blunting and increased intraepithelial lymphocytes. A small bowel aspirate was negative for bacteria. The suspicion for TS was high and the patient was started on tetracycline $250 \mathrm{mg}$ four times daily for a three-month course. Within two weeks the patient reported feeling significantly better with resolution of diarrhea, increased energy and weight gain. He then promptly left for another mission to Hawaii.

The patient returned to our clinic 2 years later stating he had stayed well, without diarrhea, abdominal pain, and weight had returned to pre-illness baseline. We repeated an EGD to evaluate the 
small bowel; the mucosa looked normal and random duodenal biopsies revealed normalization of villi and no diagnostic abnormality was present.

\section{Discussion}

Tropical sprue (TS) is an acquired intestinal malabsorption syndrome of unknown etiology that affects residents and tourists of tropical regions including West Africa, Central America, South America, the Caribbean, Puerto Rico, South East Asia and the Indian subcontinent [1]. It still accounts for almost $40 \%$ of malabsorption in adults and children in South Asia [2-5]. TS has not been described in Jamaica or sub-Saharan Africa [6]. The etiology remains elusive, but it has been suggested that persistent infection may be responsible and it is also referred to as 'post-infective tropical malabsorption' [7, 8]. Contamination of the small bowel by aerobic enteric bacteria is seen with TS patients, but no specific causal agent has ever been found $[9,10]$. Several studies have also shown increased frequency of HLA antigens Aw19 and Aw31 in Puerto Rican patients [11, 12].

Histological findings in TS may be indistinguishable from those in celiac disease which is part of the differential diagnosis for TS. However, TS involves the entire length of the small bowel while celiac disease typically spares the terminal ileum. Villous blunting is noted, but the complete villus flattening that can be seen in celiac disease is rare in TS [13-15]. Small intestine bacterial overgrowth histology may look identical to TS, however risk factors for dysmotility and stasis usually exist. Crypt hyperplasia and epithelial cell dysfunction in the proximal and distal small intestine contribute to multiple nutrient deficiencies. Reduced absorption of carbohydrates (xylose), fat, and fat-soluble vitamins is seen. Proximal small bowel involvement results in iron and folate deficiencies while terminal ileum involvement can cause vitamin B12 deficiency and bile acid malabsorption, which may further exacerbate diarrhea [5].

Symptoms of TS include chronic nonbloody diarrhea, weight loss, bloating, and abdominal cramps. Physical exam may reveal pallor, angular stomatitis, glossitis, mouth ulcers, and peripheral edema due to protein deficiency. In severe cases, vitamin A deficiency can result in night blindness, and chronic low vitamin B12 levels may cause neurologic symptoms due to subacute combined degeneration of the spinal cord.

Management begins with restoring fluid and electrolytes and repleting vitamin deficiencies. Parenteral vitamin B12, oral folate and iron supplementation can result in improvement in symptoms and weight gain even before intestinal absorption. Folate supplementation (1-5 mg daily) can result not only in improvement of the macrocytic anemia, but also in improvement in villi structure $[16,17]$. A quick and dramatic symptomatic response with folate therapy is observed so frequently in TS and so rarely in other forms of small bowel disease with megaloblastic anemia that it is considered to be diagnostic of the illness [18]. Antibiotics are commonly used with the preferred agents being tetracycline ( $250 \mathrm{mg}$ four times daily) and doxycycline (100 $\mathrm{mg}$ daily) for 3-6 months $[5,9]$. Dietary restriction on long-chain fatty acids also helps to reduce diarrhea [5]. Travellers returning to nontropical regions will generally recover completely after treatment, however relapse rates of $20-50 \%$ are seen in people permanently residing in endemic regions $[5,19]$.

A typical scenario that may be seen in the United States is that of a patient with chronic diarrhea who has lived or recently visited a tropical region. The acute illness likely started overseas and resolved within about a week, but then the patient continues to experience a milder form of persistent diarrhea with ongoing malaise and weight loss. No definite 


\begin{tabular}{r|l|l|l}
$\begin{array}{r}\text { Case Reports in } \\
\text { Gastroenterology }\end{array}$ & $\begin{array}{l}\text { Case Rep Gastroenterol 2010;4:168-172 } \\
\text { D0I: 10.1159/000314231 }\end{array}$ & Published online: May 19, 2010 & $\begin{array}{l}\text { O 2010 S. Karger AG, Basel } \\
\text { ISSN 1662-0631 } \\
\text { www.karger.com/crg }\end{array}$ \\
\hline
\end{tabular}

infectious source is identified and laboratory tests may reveal megaloblastic anemia due to folate and/or vitamin B12 deficiency. In addition, the levels of other vitamins like A and D may also be reduced. Upper endoscopy generally reveals normal mucosa, but small bowel biopsies will show an increase in intraepithelial lymphocytes and blunting of villi (total villous atrophy which can be seen in celiac disease is not a common in TS). If performed, a $100 \mathrm{~g}$ fat diet 72-hour fecal fat collection test will be abnormal in most patients.

Both of our patients presented to us with chronic nonbloody diarrhea and weight loss after having lived in parts of the world where TS is endemic. No specific infection was found in either, and although celiac disease was in the differential diagnosis, clinical, serologic, and histologic evidence did not support the diagnosis. The index of suspicion for TS should be high in such cases and a course of tetracycline and folic acid in both of our patients led to dramatic improvement relatively quickly, and remission has been maintained.

Fig. 1. Photomicrograph of case 2 of TS. The image on the left (stained with hematoxylin-eosin, magnification $100 \times$ ) shows shortened, stubby villi and modest crypt hyperplasia in the duodenum. The lamina propria shows increase in mononuclear cells. The picture on the right (same stain, magnification $400 \times$ ) shows the presence of intraepithelial lymphocytes in the superficial epithelium and identifiable plasma cells in the lamina propria.
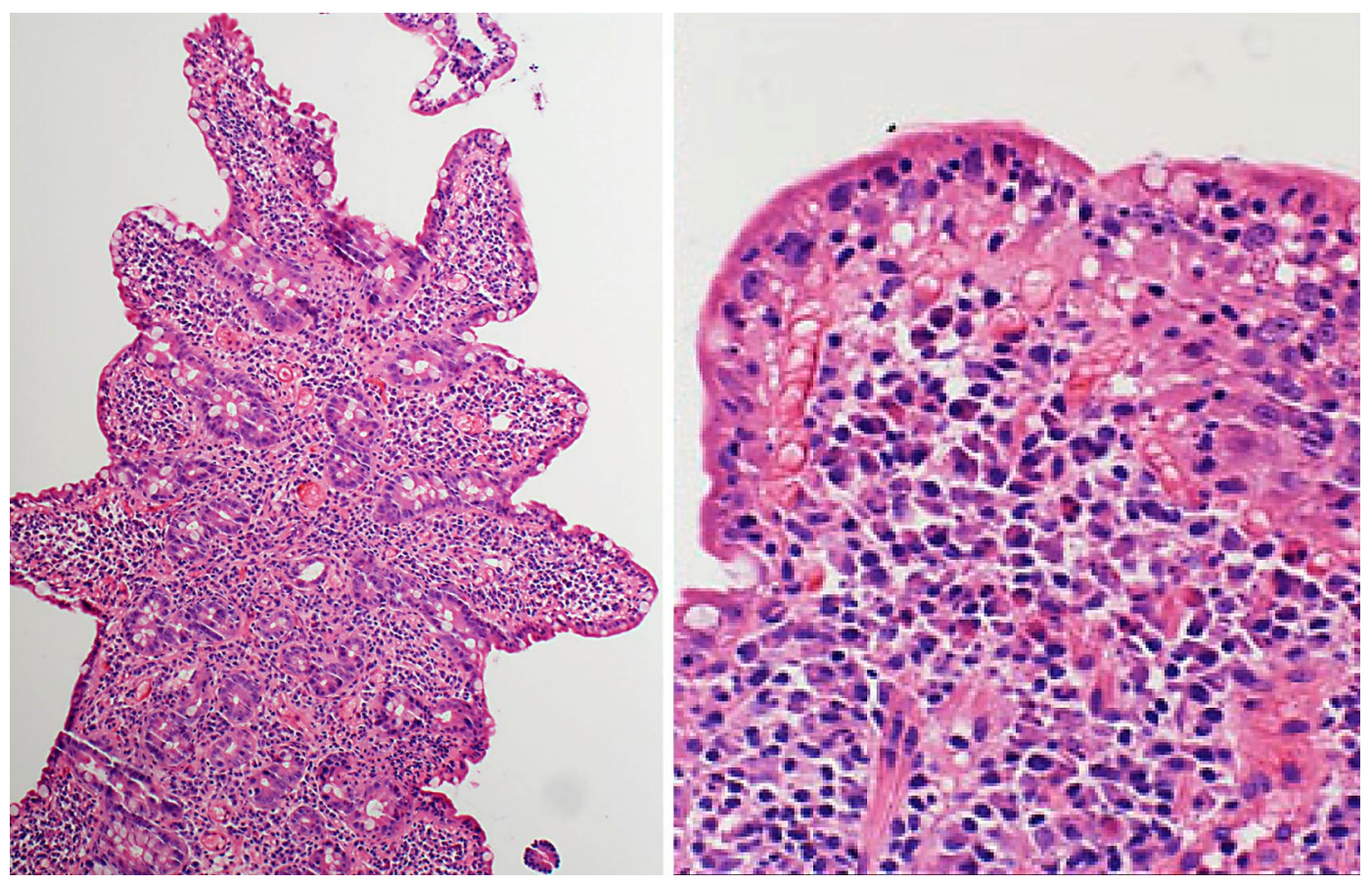


\section{References}

1 Mathan VI, Baker SJ: The epidemiology of tropical sprue; in Tropical Sprue and Megaloblastic Anaemia. Wellcome Trust Collaborative Study. London, Churchill Livingstone, 1971.

2 Khokhar N, Gill ML: Tropical sprue: revisited. J Pak Med Assoc 2004;54:133-134.

- 3 Mittal SK, Rajeshwari K, Kalra KK, Srivastava S, Malhotra V: Tropical sprue in north Indian children. Trop Gastroenterol 2001;22:146-148.

4 Baker SJ, Mathan VI: Syndrome of tropical sprue in South India. Am J Clin Nutr 1968;21:984-993.

5 Ramakrishna BS, Venkataraman S, Mukhopadhya A: Tropical malabsorption. Postgrad Med J 2006;82:779-787.

6 Lim ML: A perspective on tropical sprue. Curr Gastroenterol Rep 2001;3:322-327.

7 Tomkins AM, Drasar BS, James WP: Bacterial colonisation of jejunal mucosa in acute tropical sprue. Lancet 1975;1:59-62.

8 Cook GC: Aetiology and pathogenesis of postinfective tropical malabsorption (tropical sprue). Lancet 1984;1:721-723.

9 Walker MM: What is tropical sprue? J Gastroenterol Hepatol 2003;18:887-890.

10 Ghoshal UC, Ghoshal U, Ayyagari A, et al: Tropical sprue is associated with contamination of small bowel with aerobic bacteria and reversible prolongation of orocecal transit time. J Gastroenterol Hepatol 2003;18:540-547.

$\checkmark 11$ Menendez-Corrada R, Nettleship E, Santiago-Delpin EA: HLA and tropical sprue. Lancet 1986;2:1183-1185.

12 Sengupta S, Naik S, Naik SR: HLA antigen frequency in endemic tropical sprue. Indian J Gastroenterol 1983;2:12-13.

13 Owens SR, Greenson JK: The pathology of malabsorption: current concepts. Histopathology 2007;50:64-82.

14 Swanson VL, Thomassen RW: Pathology of the jejunal mucosa in tropical sprue. Am J Pathol 1965;46:511-551.

15 Wheby MS, Swanson VL, Bayless TM: Comparison of ileal and jejunal biopsies in tropical sprue. Am J Clin Nutr 1971;24:117-123.

$\checkmark 16$ Klipstein FA: Antibiotic therapy in tropical sprue: the role of dietary folic acid in the hematologic remission associated with oral antibiotic therapy. Ann Intern Med 1964;61:721-728.

17 Sheehy TW, Baggs B, Perez-Santiago E, Floch MH: Prognosis of tropical sprue. A study of the effect of folic acid on the intestinal aspects of acute and chronic sprue. Ann Intern Med 1962;57:892-908.

18 LaMont JT, Feldman M, Ginsburg C: Tropical Sprue. UptoDate 2009.

19 Rickles FR, Klipstein FA, Tomasini J, Corcino JJ, Maldonado N: Long-term follow-up of antibiotic-treated tropical sprue. Ann Intern Med 1972;76:203-210. 